\title{
Memory, attention, and executive function in chronic fatigue syndrome
}

\author{
Eileen Joyce, Stephen Blumenthal, Simon Wessely
}

\begin{abstract}
Objectives-To examine cognitive function in chronic fatigue syndrome.

Methods-Twenty patients with chronic fatigue syndrome recruited from primary care and 20 matched normal controls were given CANTAB computerised tests of visuospatial memory, attention, and executive function, and verbal tests of letter and category fluency and word association learning.

Results-Patients with chronic fatigue syndrome were impaired, predominantly in the domain of memory but their pattern of performance was unlike that of patients with amnesic syndrome or dementia. They were normal on tests of spatial and pattern recognition memory, simultaneous and delayed matching to sample, and pattern-location association learning. They were impaired on tests of spatial span, spatial working memory, and a selective reminding condition of the pattern-location association learning test. An executive test of planning was normal. In an attentional test, eight subjects with chronic fatigue syndrome were unable to learn a response set; the remainder exhibited no impairment in the executive set shifting phase of the test. Patients with chronic fatigue syndrome were also impaired on verbal tests of unrelated word association learning and letter fluency.

Conclusion-Patients with chronic fatigue syndrome have reduced attentional capacity resulting in impaired performance on effortful tasks requiring planned or self ordered generation of responses from memory.
\end{abstract}

\section{$(尹$ Neurol Neurosurg Psychiatry 1996;60:495-503)}

Keywords: chronic fatigue syndrome; memory; attention; cognition; effortful processing

Patients with chronic fatigue syndrome often complain of poor concentration and memory. Several studies have looked for objective evidence of cognitive dysfunction with a range of standardised neuropsychological tests but have found only mild deficits, if any. ${ }^{1-6}$ Other studies have found significant impairments but within these there is no consensus as to which cognitive processes are primarily affected. ${ }^{7-13}$ For example, Riccio et al found a discrete memory deficit, ${ }^{7}$ DeLuca et $a l^{89}$ found a selec- tive impairment in information processing speed, whereas Smith ${ }^{10}$ and Smith et al ${ }^{11}$ have shown deficits in psychomotor function and selective and sustained attention as well as wide ranging memory deficits. Two studies have examined memory and information processing while measuring evoked potentials and again the results are contradictory as one study found distinct abnormalites in the P300 cognitive evoked response ${ }^{14}$ whereas the other did not. ${ }^{15}$

One explanation of these discrepant findings concerns sample selection. Most studies have been of patients with chronic fatigue syndrome recruited in specialist settings. These patients tend to have long durations of illness and high rates of psychiatric morbidity both of which might confound neuropsychological performance and produce variable data. The role of expectancy and the controversial nature of chronic fatigue syndrome might also exert an influence. We have attempted to overcome the problem of subject selection bias by studying patients with chronic fatigue syndrome recently identified prospectively in primary care.

A second possible explanation for the discrepancies concerns differences in the sensitivity of the neuropsychological tests used, both within and between studies. For example, several of the studies have used clinical tests of memory and attention devised for the assessment of brain damaged groups of patients, which may not be sensitive to more subtle impairments. In this study we have used the Cambridge automated neuropsychological test battery (CANTAB). ${ }^{16}$ This consists of computerised neuropsychological tests shown to be sensitive to mild cognitive abnormalities in both psychiatric and neurological disorders. Because no clear hypotheses concerning the nature of the cognitive complaints in chronic fatigue syndrome can be derived from existing publications and because these complaints might be attributable to abnormalities in any one of several cognitive processes, we included several tests of memory, attention, and executive function. Finally, because these tests are all visual in nature, we also employed two verbal tasks for comparative purposes.

\section{Methods}

PATIENTS

All patients were identified at the final stage of a large prospective community based study of chronic fatigue syndrome and its relation to viral infection. ${ }^{1718} \mathrm{~A}$ total of 2376 patients 
aged 18 to 45 were recruited from primary care after a consultation with a general practitioner for a viral infection. Of these, 1985 were followed up at six months and those scoring above a predefined cut off on a scale measuring fatigue ${ }^{19}$ completed several questionnaires to establish the criteria for chronic fatigue syndrome and underwent a standardised psychiatric assessment. ${ }^{20}$ Out of the original 2376, 36 were identified as fulfilling the current United Kingdom criteria for chronic fatigue syndrome. ${ }^{21}$ These patients were spread across five general practices in the south of England. Twenty nine were living in two practices close to our research team and these were invited to participate. Twenty patients agreed to be tested. Of those not tested, one refused and the remainder failed to reply. Compared with the group of patients with chronic fatigue syndrome tested, there were more men among the patients not tested but no differences in severity of disease. Controls were selected from the same cohort study and were 20 patients who had originally consulted with a viral infection but were not chronically fatigued six months later. Most of the fatigued patients did not consider themselves to have chronic fatigue syndrome or myalgic encephalomyelitis and were not seeking help under these labels.

Table 1 shows that the two groups were matched for age, sex ratio, and WAIS-R IQ estimated from the national adult reading test (NART). ${ }^{22}$ Patients were asked to complete a 13 item fatigue questionnaire covering items relating to both physical and mental fatigue ${ }^{19}$ and the hospital anxiety and depression scale ${ }^{23}$, a well validated questionnaire which avoids using items concerning somatic symptoms including fatigue. A score of 11 or more designates pathological anxiety or depression. Table 1 shows the scores on these scales. The tests were given in the same order for each subject and the test session took about three hours to complete. All controls and 15 patients with chronic fatigue syndrome completed the tests in one session. The remaining patients performed the tests over two sessions because the development of subjective fatigue curtailed the first session. Because of the long test session, the possibility that the results could be explained entirely by increased fatigue developing in the chronic fatigue syndrome group compared with controls as the test session progressed was considered. Accordingly, the performance of the groups with respect to the order of test was

Table 1 Group mean (SEM) for age, IQ estimated from the national adult reading test (NART), self ratings of physical and mental fatigue, hospital anxiety and depression scale self ratings of depression, and anxiety in normal controls and subjects with chronic fatigue syndrome (CFS)

\begin{tabular}{llll}
\hline & Controls & \multicolumn{1}{l}{ CFS } & P value \\
\hline No & \multicolumn{1}{l}{ 20 } & \multicolumn{1}{c}{20} & \\
Sex ratio (F/M) & $16 / 4$ & $17 / 3$ & NS \\
Age & $34.90(1.07)$ & $37.20(1.36)$ & NS \\
NART & $118.36(2.36)$ & $113.80(1.99)$ & NS \\
Fatigue & $0.90(0.26)$ & $2.05(0.38)$ & $<0.001$ \\
$\quad$ Mental & $1.95(0.52)$ & $5.45(0.50)$ & $<0.02$ \\
Physical & $2.90(0.72)$ & $7.40(0.80)$ & $<0.001$ \\
Depression & $6.45(0.75)$ & $9.95(1.25)$ & 0.02 \\
Anxiety & & & \\
\hline
\end{tabular}

examined. There was no evidence that the patients with chronic fatigue syndrome were more impaired on tests performed towards the end of the test session. In fact, the most significant group differences were found on tests of spatial span and spatial working memory, which were performed early in the session.

\section{PROCEDURE}

\section{Computerised tests}

The CANTAB battery runs on a personal computer with an Intersolve LCD portable touch sensitive screen. Detailed descriptions of these tests have been published elsewhere (see ${ }^{16293436}$ ) and only a brief outline is given below.

Pattern recognition memory-Subjects were shown 12 visual patterns one after another. They were then shown 12 successive pairs of patterns of which one had been shown before and the other was novel. They were asked to touch the familiar pattern. The procedure was then repeated with 12 new patterns (maximum score $=24$ ).

Spatial recognition memory-Subjects were shown five squares, one at a time, at different locations on the screen. They were then shown five successive pairs of squares of which one was at a previous location and the other was at a novel location. They were asked to touch the square at the location previously used. Four blocks of five stimuli were presented in total (maximum score $=20$ ).

Simultaneous and delayed matching to sampleSubjects were shown a complex abstract pattern and then asked to pick out this pattern from an array of four similar patterns. In the simultaneous condition, the sample pattern remained on the screen while the choice stimuli appeared. In the delayed condition, the sample pattern disappeared and the choice patterns appeared after a delay of 0,4 , or 12 seconds. There were 10 test trials at each of the simultaneous and delay conditions, which were presented in a randomised order.

Paired associates learning-Subjects were required to learn the location of nine sets of patterns individually placed in boxes on the screen. For each set, the subject was allowed a maximum of 10 trials to learn the pattern-location associations. In the first eight sets, the number of patterns presented varied from one to eight. The ninth set consisted of eight patterns but, instead of the subject being reminded of the location of all patterns on each trial, only the location of patterns incorrectly located on the previous trial were reshown. Therefore, in this selective reminding set, subjects had to maintain the memory for correctly learned pattern-location associations while learning the correct locations of patterns which they had failed to locate in the previous trial.

Spatial span-In this computerised version of Corsi's block tapping task, ${ }^{24}$ the subjects watched a sequence of white squares on the screen change colour one at a time. They were then asked to reproduce this sequence. After a correct response, the length of the sequence increased by one square up to a maximum of nine. 
Spatial working memory task-Subjects were asked to search through a number of boxes presented on the screen to find a token. The key instruction was that once a token had been found within a box then that box would not be used again to hide a token during that particular trial. On each trial, the total number of blue tokens to be found corresponded to the number of boxes on the screen. There were four test trials with each of three, four, six, and eight boxes.

Planning-This task is closely related to the Tower of London task developed by Shallice and McCarthy. ${ }^{25}$ Two sets of three coloured "balls" were presented on the screen. The subjects were asked to rearrange the balls in the bottom display such that their positions matched the "goal" arrangement in the top half of the screen. The starting position of the balls was varied such that in any particular trial the solution could only be reached after a minimum of two, three, four, or five moves. Subjects were instructed to examine the position of the balls at the beginning of each problem and attempt to solve it in the minimum possible number of moves. The program stored the number of moves required by the subject to rearrange the balls and measured the selection and movement latencies for both the first and the subsequent moves. For each test problem a "yoked control" condition was employed to provide a baseline measure of motor initiation and execution times in which subjects were required to follow a sequence of single moves executed by the computer in the top half of the screen by moving the corresponding ball in the lower arrangement. The test was yoked to the main test in the sense that in each trial, the movement of the balls was an exact replication of those moved by the subject in the corresponding test trial.

Visual search and matching to sample task-A central red box surrounded by eight white boxes appeared on the screen. To initiate each trial, the subjects were required to depress a key pad after which the boxes opened to disclose the central target stimulus surrounded by choice stimuli among which an identical match had to be located and touched. On equal proportions of the 48 trials there were one, two, four, or eight different patterns to choose from.

Attentional set shifting task-In this task, subjects were trained on a series of visual discriminations and reversals which varied in two perceptual dimensions, one of which was correct or relevant and one of which was incorrect or irrelevant. At first, they were presented with two purple shapes and asked to guess which was correct by touching it. The computer provided feedback as to whether the response was correct or incorrect. After six correct responses the alternative pattern then became correct. A second dimension, white lines, was then introduced to produce compound patterns and the subjects underwent compound discrimination learning and reversal stages in which they were required to respond to the same relevant dimension and ignore the irrelevant dimension. The next two stages required the subjects to transfer the rule that they had learned to a new set of exemplars. These were the intradimensional shift and reversal stages. In the final two stages, extradimensional shift and reversal, the subjects were required to shift attention from the previously relevant dimension to the previously irrelevant dimension. For each stage, continuation to the next one was dependent on a criterion of six successive correct reponses being reached. If criterion was not reached by the 50 th trial of a stage, then the test was discontinued and subjects did not proceed to the next stage.

\section{Pencil and paper tests}

In the word association learning subtest of the Wechsler memory scale revised, ${ }^{26}$ subjects were read a list of eight word pairs. The first word of each pair was then presented and the subject was required to give the associated word. Half of the word pairs were semantically relatedfor example, east-west-and half were unrelated-for example, dark-crush. The subjects were given three trials and scored separately for "easy" (maximum 12) and "hard" (maximum 12) associates.

Subjects were also given two tests of verbal fluency. Firstly, they were asked to produce as many words as they could think of beginning with the letters $F, A$, and $S$ for one minute each (FAS verbal fluency). Secondly, they were asked to produce items belonging to the categories, fruit and vegetables, occupations, and animals for one minute each.

\section{STATISTICAL ANALYSIS}

This was performed using the statistical package for social scientists (SPSS PC). For most of the dependent variables a univariate analysis of variance (ANOVA) was applied with repeated measures on the second factor for two factor designs. Logarithmic (base 10) transformations were applied to latency data and arc sine transformations were applied for the analysis of proportional data to reduce skew. For the attentional set shifting task, the data for the number of subjects passing and failing were cast into contingency tables and analysed using the likelihood ratio method which is suitable for small cell frequencies. ${ }^{27} 28$ The resulting information statistic (2i) is distributed as $\chi^{2}$. Correlations between scores on neuropsychological tests and ratings of depression, anxiety, and fatigue were analysed using Spearman's test.

\section{Results}

The chronic fatigue syndrome group rated themselves as significantly more fatigued than controls (table 1) and this was true for both physical $(F(1,38)=23.52 ; \mathrm{P}<0.001)$ and mental fatigue $(F(1,38)=6 \cdot 22 ; P=0.017)$. Similarly the chronic fatigue syndrome group rated themselves as more anxious $(F(1,37)=$ $5 \cdot 91, \mathrm{P}=0.02)$ and more depressed $(F(1,37)$ $=17 \cdot 70 ; \mathrm{P}<0.001)$ than controls. However, neither the anxiety nor the depression ratings for the chronic fatigue syndrome group surpassed the cut off score for significant psychopathology. 
Table 2 Mean (SEM) CANTAB neuropsychological variables for groups of normal controls and subjects with chronic fatigue syndrome (CFS)

\begin{tabular}{|c|c|c|c|}
\hline & Controls & CFS & Pvalue \\
\hline $\begin{array}{l}\text { Pattern recognition: } \\
\text { Number correct (max 24) } \\
\text { Latency correct (s) }\end{array}$ & $\begin{array}{r}20 \cdot 65(0 \cdot 84) \\
2 \cdot 24(0 \cdot 13)\end{array}$ & $\begin{array}{r}20 \cdot 75(0 \cdot 76) \\
2 \cdot 39(0 \cdot 13)\end{array}$ & $\begin{array}{l}\text { NS } \\
\text { NS }\end{array}$ \\
\hline $\begin{array}{l}\text { Spatial recognition: } \\
\text { Number correct }(\max 20) \\
\text { Latency correct }(s)\end{array}$ & $\begin{array}{r}16 \cdot 15(0 \cdot 48) \\
2 \cdot 42(0 \cdot 20)\end{array}$ & $\begin{array}{r}15.45(0.46) \\
2.68(0.25)\end{array}$ & $\begin{array}{l}\text { NS } \\
\text { NS }\end{array}$ \\
\hline $\begin{array}{l}\text { Matching to sample: } \\
\text { Simultaneous } \\
\text { Number correct }(\max 10) \\
\text { Latency correct } \\
\text { Delayed }\end{array}$ & $\begin{array}{l}9 \cdot 65(0 \cdot 13) \\
2 \cdot 66(1 \cdot 18)\end{array}$ & $\begin{array}{l}9 \cdot 55(0 \cdot 11) \\
2 \cdot 87(2 \cdot 23)\end{array}$ & $\begin{array}{l}\text { NS } \\
\text { NS }\end{array}$ \\
\hline $\begin{array}{l}\text { Number correct }(\max 30) \\
\text { Latency correct }\end{array}$ & $26.05(0.61)$ & $24 \cdot 25(0 \cdot 69)$ & $\begin{array}{l}<0.06 \\
\text { gp } \times \text { delay: } \\
<0.05\end{array}$ \\
\hline $\begin{array}{r}0 \mathrm{~s} \\
4 \mathrm{~s} \\
12 \mathrm{~s}\end{array}$ & $\begin{array}{l}3.07(0 \cdot 20) \\
3 \cdot 28(0 \cdot 16) \\
3 \cdot 86(0 \cdot 20)\end{array}$ & $\begin{array}{l}2 \cdot 74(0 \cdot 19) \\
2 \cdot 23(0 \cdot 21) \\
4 \cdot 25(0 \cdot 33)\end{array}$ & $\begin{array}{l}\text { NS } \\
\text { NS } \\
\text { NS }\end{array}$ \\
\hline $\begin{array}{l}\text { Paired associates: } \\
\text { Sets completed (max 9) } \\
\text { Memory score } \\
\text { First trials correct }\end{array}$ & $\begin{array}{r}8 \cdot 90(0 \cdot 10) \\
24 \cdot 70(1 \cdot 20) \\
6 \cdot 15(0 \cdot 22)\end{array}$ & $\begin{array}{r}8.90(0.07) \\
23.50(1.16) \\
6.00(0.25)\end{array}$ & $\begin{array}{l}\text { NS } \\
\text { NS } \\
\text { NS }\end{array}$ \\
\hline Spatial span & $6 \cdot 20(0 \cdot 24)$ & $5 \cdot 16(0 \cdot 26)$ & $<0.01$ \\
\hline $\begin{array}{l}\text { Spatial working memory: } \\
\text { Strategy score }\end{array}$ & $13.55(1 \cdot 21)$ & $17 \cdot 26(1 \cdot 26)$ & $<0.05$ \\
\hline $\begin{array}{l}\text { Planning: } \\
\text { Total solutions ( } \max 12) \\
\text { Perfect solutions }(\max 12) \\
\text { Excess moves }\end{array}$ & $\begin{array}{r}11.85(0.15) \\
8.95(0.52) \\
2.84(0.66)\end{array}$ & $\begin{array}{r}11.90(0.10) \\
7.85(0.44) \\
3.91(0.55)\end{array}$ & $\begin{array}{l}\text { NS } \\
\text { NS } \\
\text { NS }\end{array}$ \\
\hline $\begin{array}{l}\text { Unrelated word associations: } \\
\text { Number correct ( } \max 12)\end{array}$ & $8.95(0.51)$ & $6.85(0.59)$ & $<0.01$ \\
\hline $\begin{array}{l}\text { Verbal fluency: } \\
\text { FAS } \\
\text { Category }\end{array}$ & $\begin{array}{l}46 \cdot 95(2 \cdot 72) \\
60 \cdot 75(2 \cdot 81)\end{array}$ & $\begin{array}{l}37 \cdot 35(2 \cdot 04) \\
53 \cdot 90(2 \cdot 35)\end{array}$ & $\begin{array}{l}<0.01 \\
<0.07\end{array}$ \\
\hline
\end{tabular}

PATTERN AND SPATIAL RECOGNITION MEMORY For each task, group comparisons were made between choice accuracy and latency to make a correct choice. Table 2 shows that the two groups performed equally well on these tasks with respect to both measures $(F(1,38)$ range $0 \cdot 008-1 \cdot 125$; NS).

\section{SIMULTANEOUS AND DELAYED MATCHING TO SAMPLE}

Group comparisons were made for choice accuracy and latency to make a correct choice as shown in table 2 . For the simultaneous condition, there were no group differences for either measure $(F(1,38) \mathrm{P}<1 \cdot 0$; NS). For the delay conditions, there was a non-significant tendency for the chronic fatigue syndrome group to be less accurate independent of delay (group: $F(1,38)=3.79 ; \mathrm{P}=0.06$; group $\times$ delay: $F(2,76)=2 \cdot 20 ; \mathrm{NS})$. There was a significant effect of delay $(F(2,76)=3 \cdot 44$; $P<0.05)$ indicating that both groups made more errors at the longer delays. The group effect for latency was not significant $(F(1,38)$ $=0.11 ; \mathrm{NS})$ but there was a significant effect of delay $(F(2,76)=46.85 ; \mathrm{P}<0.001)$ and $\mathrm{a}$ group by delay interaction $(F(2,76)=3 \cdot 28$; $P<0.05)$. Main effects analysis showed that there were no significant group differences at any delay $(F(1,38)$ range $0.53-1.53 ; \mathrm{NS})$. Individual group analyses of the delay effect indicated that both groups took longer to respond as the delay increased (control: $F(2,38)=15.41 ; P<0.001$; chronic fatigue syndrome: $F(2,38)=31.88 ; \mathrm{P}<0.001)$. Inspection of the data disclosed that there was a cross over in latency scores so that the chronic fatigue syndrome groups were faster that con- trols at 0 seconds delay, the same at 4 seconds, and slower at 12 seconds (table 2).

\section{PAIRED ASSOCIATE LEARNING}

Performance was assessed by four measures (table 2 and fig 1). Firstly, most subjects in each group were successful in learning all pattern-locations in each set, including those presented on the selective reminding set $(F(1,38)$ $=0.01$; NS). Secondly, a memory score, calculated as the total number of patterns located correctly after the first presentation summed across sets, was no different between the groups $(F(1,38)=0.52 ; \mathrm{NS})$. Thirdly, the number of completely correct sets of associations after the initial presentation (first trial corrects) was not different between the groups $(F(1,38)=0 \cdot 20$; NS). The fourth measure examined learning efficiency calculated as the number of trials required to complete each stage (fig 1). There were no group differences in trials taken at the six pattern $(F(1,38)=$ 0.35 ; NS) and eight pattern $(F(1,38)=3.63$; NS) sets. However, the patients with chronic fatigue syndrome took significantly more trials to learn the final set of eight associations in which subjects were only shown the correct position of incorrect responses after each trial $(F(1,38)=5.63 ; P=0.023)$. There was a significant group by trials interaction $(F(9,342)=2.17 ; \quad P=0.024)$ and main effects analysis showed significant differences in performance on trials 2,4 , and $5(F(1,38)$ range 5.26-7.150; all $P<0 \cdot 05$ ).

\section{SPATIAL SPAN}

The groups were compared in terms of the number of squares that were touched in the correct serial order (table 2). The chronic fatigue syndrome group was significantly impaired on this measure $(F(1,37)=8.95 ; \mathrm{P}$ $=0.005)$.

SPATIAL WORKING MEMORY

In this task two types of search error are possible. Firstly, a subject may return to open a box in which a token has already been found (a

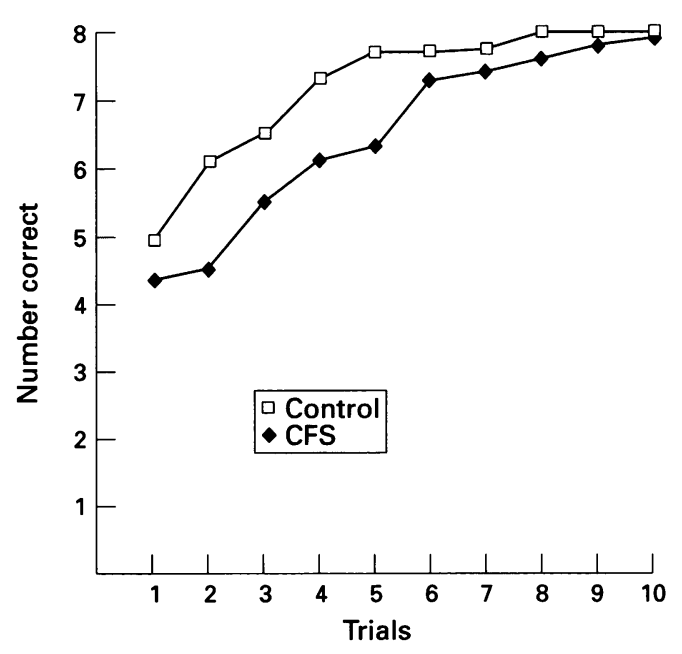

Figure 1 Performance on the final stage of the paired associate learning task for patients with chronic fatigue syndrome $(C F S, n=20)$ and normal controls $(n=20)$. 


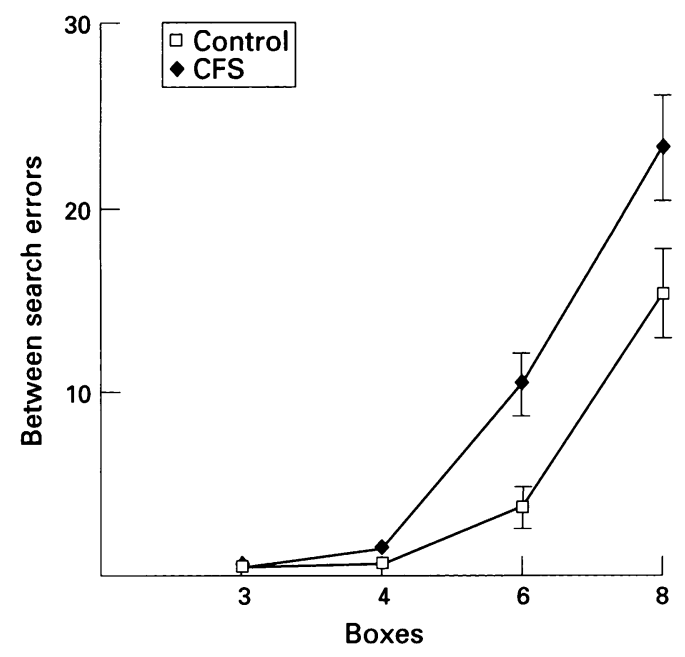

Figure 2 Between-search errors at each stage of the spatial working memory task for subjects with chronic fatigue syndrome (CFS, $n=20$ ) and normal controls $(n=20)$.

"between-search" error). Secondly, a subject may return to a box already opened in the same search sequence (a "within search" error). Figure 2 shows that the chronic fatigue syndrome group made significantly more between-search errors (group: $F(1,37)=$ $7.52 ; P=0.009$ ). There was a significant effect of difficulty-that is, number of boxes, $(F(3,111)=85.81, \mathrm{P}<0.001)$ and a significant group by difficulty interaction $(F(3,111)$ $=4.7 ; \quad \mathrm{P}=0.004)$. Main effects analysis showed that the chronic fatigue syndrome group were specifically worse than controls at the six box $(F(1,37)=11 \cdot 17 ; P=0.002)$ and eight box $(F(1,37)=4.43 ; \mathrm{P}=0.04)$ stages. As there were very few within search errors at any level of difficulty these were summed across trials. There was no difference between the groups on this measure $(F(1,37)$ $=0.21 ; \mathrm{NS})$. As well as number of errors, the subjects were scored on a search strategy on a scale of $1-37$. A perfect strategy score of 1 is obtained when the same box is used to initiate each search sequence within the six and eight box problems. Table 2 shows that the chronic fatigue syndrome group was significantly inferior in the use of this search strategy $(F(1,37)$ $=4.55 ; \mathrm{P}=0.04)$. The use of a strategy was related to performance as covariance for strategy rendered the group difference in between search errors non-significant $(F(1,36)=2 \cdot 58$; $\mathrm{P}=0 \cdot 12$ ) and, for both groups, strategy score correlated with between search errors combined for six and eight box stages (control: $r=$ $0.78 ; \mathrm{P}<0.001$; chronic fatigue syndrome: $0.81 ; P<0.001)$. Spatial span also affected performance as entering this as a covariate also rendered the group difference between-search errors non-significant $(F(1,36)=1 \cdot 80 ; \mathrm{P}=$ $0 \cdot 19)$. For the chronic fatigue syndrome group, spatial span correlated with strategy score $(r=-0.60 ; \mathrm{P}=0.007)$ and there was a trend towards a correlation between span and between-search errors $(r=-0.41 ; \mathrm{P}=$ $0.08)$. For controls, spatial span correlated with between-search errors $(r=-0.68 ; \mathrm{P}=$ $0.001)$ and there was a trend towards a signifi- cant correlation with strategy score $(r=$ $-0.40 ; P=0.09$ ).

PLANNING

Performance was assessed by three measures of accuracy (table 2). Firstly, the total number of problems solved correctly was no different between the groups $(F(1,38)=0.08 ; \mathrm{NS})$. Secondly, the mean number of moves above the minimum possible, which provides a general measure of planning efficiency, was no different between the groups $(F(1,38)=1 \cdot 7$; NS). Finally, the proportion of problems solved in the minimum number of moves, which provides a more specific measure of efficient planning ability, was also no different between the groups $(F(1,38)=2 \cdot 65 ; \mathrm{NS})$. As expected, there was a significant effect of problem difficulty for all measures $(F(3,114)$ range $32 \cdot 16-617 \cdot 3$; all $\mathrm{P}<0.001)$ but there were no significant interactions between group and difficulty $(F(3,114)$ range $0 \cdot 08-1 \cdot 22$; NS)

Baseline measures of motor initiation and motor execution times were extracted from the 12 yoked control trials. The motor initiation time was the mean time between the onset of each problem and the completion of the first touch (correct touch of the required ball). The motor execution time was the time between touching the first ball and completing the sequence of required moves that comprised the whole problem. The chronic fatigue syndrome group was slower on both of these measures across all levels of difficulty as indicated by significant group effects and nonsignificant interaction terms. This finding was true for all problems solved and for those solved in the minimum number of moves possible $(F(1,38)$ range $5 \cdot 35-11 \cdot 45$; $\mathrm{P}=0.002-0.026)$.

The motor initiation and execution times were used to derive estimates of planning or thinking times in the main task. Two main estimates were calculated. The initial thinking time was the interval between the presentation of the problem and the first touch of the ball minus the corresponding motor initiation time. There were no differences between the groups on this measure whether assessed for all problems solved or only those solved in the minimum number of moves possible $(F(1,38)$ $=0.08$ and 0.01 respectively; NS). The subsequent thinking time was the time between the selection of the first ball and the completion of the problem minus the motor execution time derived from the corresponding control problem. Because this measure varied with problem length, scores were divided by the number of moves taken to complete the solution to give an estimate of the average thinking time per move. Again there were no differences between the groups whether measured across all problems solved or those solved in the minimum number of moves $(F(1,38)=1.31$ and 0.38 respectively; NS). For all measures of latency, except subsequent following time for perfect solutions, there was a signficant effect of problem difficulty showing that latency to respond increased for 
both groups as the problems became more difficult $\quad(F(3,114)$ range: 3.87-108.8; $\mathrm{P}<0.01-\mathrm{P}<0.001)$.

VISUAL SEARCH AND MATCHING TO SAMPLE There were no differences between the groups as measured either by the number of correct responses over levels of difficulty-that is, increasing number of choice stimuli (group: $F(1,36)=0.52$; NS: group $\times$ difficulty: $F(3,108)=1 \cdot 26$; NS) or by latency to touch the correct stimulus (group: $F(1,36)=1 \cdot 38$; NS: group $\times$ difficulty $(F(3,108)=0.37 ; \mathrm{NS})$. Over the four levels of difficulty the control group score was 46.7 (97\% correct) and the chronic fatigue syndrome group score was 47 ( $98 \%$ correct). As more choices were introduced, latency measures increased from a mean of 1.53 seconds to 3.93 seconds for the controls and 1.74 seconds to 4.26 seconds for the chronic fatigue syndrome group. For both choice accuracy and latency measures there was a significant effect of difficulty (correct responses: $\quad(F(3,108)=17.35 ; \quad \mathrm{P}<0.001$; latency correct: $F(3,108)=369.9 ; \mathrm{P}<0.001)$ showing that both groups tended to make more errors and took longer to respond as a function of the number of choices.

\section{ATTENTIONAL SET SHIFTING TASK}

Twelve patients with chronic fatigue syndrome $(60 \%)$ and $19(95 \%)$ controls successfully completed all nine stages of the task $(2 \mathrm{i}=$ $7 \cdot 79 ; \mathrm{df}=1 ; \mathrm{P}<0.01)$. Cumulative analyses at each stage showed that up to and including the compound reversal stage, three patients with chronic fatigue syndrome had dropped out compared with none of the controls $(2 \mathrm{i}=$ 4.40 ; df $=1 ; \mathrm{P}<0.05)$. The remaining subjects successfully passed the intradimensional shift stage. Three patients with chronic fatigue syndrome and one control failed at the intradimensional reversal stage and two patients with chronic fatigue syndrome failed the extradimensional shift stage. The cumulative analysis remained significant at each of these stages. However, when the analysis was restricted to those actually attempting each stage, there were no significant differences between the groups at any stage. To examine efficiency of performance at the critical extra dimensional shift stage, the number of trials to criterion and the number of errors for those attempting the extra dimensional shift stage was analysed. Neither of these measures were significant $(F(1,29)=0.67$ and 1.74 respectively; NS).

\section{WORD ASSOCIATION LEARNING}

There was no difference between the groups in learning the related word associations, both scoring over $90 \%$ correct. Table 2 shows that the chronic fatigue syndrome group learned fewer unrelated associations than the controls $(F(1,38)=7 \cdot 30 ; \mathrm{P}=0.01)$.

\section{VERBAL FLUENCY}

Table 2 shows that the chronic fatigue syndrome group produced significantly fewer words in the letter fluency task $(F(1,38)=$ $8.00 ; \quad P=0.008)$. Although the chronic fatigue syndrome group also produced fewer category words, this failed to reach signficance $(F(1,38)=3.51 ; \mathrm{P}<0.07)$

\section{CORRELATIONS}

Spearman's correlations were performed between total fatigue score, depression and anxiety self ratings, and neuropsychological performance for control and chronic fatigue syndrome groups separately. The neuropsychological scores entered were: spatial span, spatial working memory strategy score and combined between-search errors at the six and eight box stage, planning task perfect solutions, paired associate learning total correct responses at the selective reminding stage, the stage passed on attentional set shifting task, verbal fluency scores for letter and category, and unrelated word associate learning score. There were no significant correlations between clinical measures of fatigue, anxiety, and depression and any of the neuropsychological measures for controls. For the chronic fatigue syndrome group, fatigue correlated with spatial recognition memory $(r=-0.57 ; \mathrm{P}=$ $0.009)$ and FAS verbal fluency $(r=0.54 ; \mathrm{P}$ $=0.015$ ). Therefore increasing fatigue was related to decreased spatial recognition memory and FAS fluency.

\section{Discussion}

In this study we have examined the cognitive function of a group of patients with chronic fatigue syndrome prospectively identified in a primary care setting. Previous studies have been based on highly selected samples with long durations of illness, high rates of psychiatric morbidity, and often intense disease attributions. Most cases in the present study were not seeking help for chronic fatigue syndrome, and few used terms such as chronic fatigue syndrome, myalgic encephalomyelitis, or post viral fatigue to describe their illness. Thus we believe that the current sample is relatively free from many biases that have influenced previous studies of neuopsychological functioning and chronic fatigue syndrome.

The patients with chronic fatigue syndrome were most impaired on tests of spatial span and spatial working memory. On the second test, there were no differences in within-search errors indicating that the patients with chronic fatigue syndrome were as proficient as controls in monitoring boxes searched within a trial. However, patients with chronic fatigue syndrome made more between-search errors than controls showing that they had difficulty in keeping track of boxes which had contained tokens on previous trials. They were also deficient in using a search strategy. Normally there is a positive correlation between strategy score and between search errors ${ }^{29} 30$ and the preservation of this relation in the chronic fatigue syndrome group suggests that the poor use of a strategy contributed to their impaired performance. This is also suggested by the finding that the group difference disappeared when strategy score was covaried with between search errors. Accurate performance on this 
task also depends upon the ability to remember the location of up to eight tokens. Thus impaired spatial span might have also contributed to the poor performance of the chronic fatigue syndrome group. This is supported by the findings that the group difference in between-search errors disappeared when spatial span was used as a covariate and that the correlational analysis showed a relation between spatial span and performance for both groups. The impairment of patients with chronic fatigue syndrome on these tests contrasts with their normal performance on the spatial recognition memory task. Thus the deficits in spatial span and spatial working memory cannot be explained by a global spatial memory deficit.

The poor strategy score of the fatigued patients on the spatial working memory task might indicate that patients with chronic fatigue syndrome have a general impairment in executive tasks requiring the ability to plan and execute reponses effectively. This is not the case, however, as patients with chronic fatigue syndrome were as accurate as controls and had similar thinking times on a specific task of planning. Although it is possible that the dissociation of performance on these two tasks is because the planning task is less sensitive to the cognitive impairment in chronic fatigue syndrome, this does not seem to be the case. The impairment of patients with chronic fatigue syndrome on the spatial working memory task seems similar in degree to that of other neurological patients who also have impaired planning, ${ }^{29} 3031-33$ once the effect of age on performance is taken into account. ${ }^{35} \mathrm{~A}$ critical difference between these two tasks is that the spatial working memory task has a much more significant mnemonic component. Thus in this study, patients with chronic fatigue syndrome had particular difficulty when required to generate planned or self ordered responses from memory as in the spatial span and spatial working memory tasks.

The extradimensional shift stage of the attentional set shifting task is formally analogous to the Wisconsin card sorting task and examines a different aspect of executive function to the planning task. There were no differences in the performance of patients with chronic fatigue syndrome and control subjects attempting this stage. There was a significant attrition of patients with chronic fatigue syndrome earlier in the test, which suggests that some patients with chronic fatigue syndrome had difficulty in learning and maintaining a response set in which attention has to be focused on a relevant dimension. A post hoc comparison of the patients who failed the test with those who completed all stages, showed that the only difference was that the first were significantly older by a mean of six years.

The patients with chronic fatigue syndrome were not impaired on a second attentional task requiring visual search and matching to sample, both groups being almost totally accurate and equally as fast on the more discriminating latency measure. It is unlikely that the results on these two tests reflect differences in task sensitivity as a different study has found the opposite result in depressed patients (Elliott, Sahakian, Robbins, McKay, Herrod, and Paykel, unpublished data). These patients were able to complete the attentional set shifting task yet were less accurate and slower than controls on a similar matching to sample task. Thus the impairment of the subgroup of patients with chronic fatigue syndrome on the set shifting task does not seem to reflect a more global problem with focused attention.

In addition to the spatial recognition memory task, patients with chronic fatigue syndrome performed well on other recognition memory tasks. On the simultaneous and delayed matching to sample task, there were no significant group differences in response accuracy. Regarding latency, although the interaction term was significant, there was no difference between the groups at any of the individual delays and both groups showed significant time courses. Thus performance on this task does not support a pattern recognition memory deficit in the chronic fatigue syndrome group and this is substantiated by the normal chronic fatigue syndrome performance on the two choice pattern recognition memory task.

The patients with chronic fatigue syndrome also performed normally according to several measures of memory and learning on the paired associates test. However, a learning deficit was seen when, on each trial, they were shown only the correct position of those stimuli that they had failed to locate on the previous trial. Because subjects were required to locate all patterns on each trial, the patients with chronic fatigue syndrome were impaired only when they had to retain some pattern locations in memory while learning others.

The deficits exhibited by the chronic fatigue syndrome group were not confined to the visual modality. Although patients with chronic fatigue syndrome were normal at learning semantically related word associations, they were impaired when the word pairs were not related. They also showed impaired verbal fluency and this was more pronounced when they were instructed to generate words beginning with a particular letter compared with words belonging to a particular category.

In summary, we have shown that patients with chronic fatigue syndrome have cognitive deficits predominantly in the domain of memory. However, they performed normally on recognition memory tests and on a graded pattern-location association learning task with the exception of the final stage. Their mnemonic difficulty became apparent on tasks requiring the planned or self ordered sequencing of responses from memory. This pattern of deficit on the CANTAB tests is unlike that seen in amnesic patients with Korsakoff's syndrome, ${ }^{30}{ }^{35}$ early Alzheimer's disease, ${ }^{36-39}$ and medial temporal lobe excisions ${ }^{42}$ as these are impaired on all memory tasks, fail early on tasks, and are not helped by strategy. Thus it seems unlikely that the problems of the patients with chronic fatigue syndrome in this study were secondary to a direct dysfunction 
of the neural substrates mediating memory. Rather, the profile of deficits suggests an attentional problem of the type set out by Hasher and Zacks. ${ }^{41}$ In their cognitive framework, encoding and retrieval memory processes occupy a position along an automatic-effortful continuum according to attentional requirements. In this study, spatial working memory, spatial span, and the paired associate selective reminding tasks, can be considered more effortful than the initial learning sets of the paired associates task and the delayed matching to sample tasks and spatial and pattern recognition tasks. This is because, in the recognition tasks memory is prompted by a stimulus cue whereas in the sample tasks, more active processes such as rehearsal, mnemonic techniques, or other strategies are required for accurate recall. The results of the traditional neuropsychological tests can also fit the same model. Thus the differential chronic fatigue syndrome performance on the word association test can be explained by learning unrelated word pairs being more effortful than learning semantically related words. Similarly, production of words belonging to a certain category may require less effort than words beginning with an initial letter because the structure of semantic memory lends itself to categorical clustering, thus explaining why the patients with chronic fatigue syndrome were more impaired than controls on the initial letter test. This is reinforced by the finding of an inverse relation between FAS fluency and fatigue ratings in patients with chronic fatigue syndrome.

Within this model, anything which reduces attentional capacity such as age, arousal, mood, and illness has been shown to impair performance on effortful tasks. ${ }^{41}$ Thus we propose that in chronic fatigue syndrome, the subjective experience of both central fatigue and impaired concentration and memory is a reflection of reduced attentional capacity which is manifest objectively as impaired performance on effortful memory tasks. Consistent with this is the possibility that the impairment of the subgroup of patients with chronic fatigue syndrome in learning a response set may be effortful because of a combination of chronic fatigue syndrome and older age.

The subjective complaints of cognitive impairment are often attributed to depression on the grounds that depression is common in chronic fatigue syndrome and is itself associated with cognitive impairment. Interestingly, some workers ${ }^{41-43}$ have argued that cognitive deficits in depression reflect reduced attentional capacity although other explanations abound. ${ }^{44}$ To explore the relation between depression and chronic fatigue syndrome, some studies have directly compared these two sets of patients on the same cognitive tests but the findings are inconclusive. ${ }^{561316}$ Others have looked for associations between depressive symptoms and cognitive function within a group of patients with chronic fatigue syndrome but only one has shown such a link. ${ }^{45}$ In our study, patients with chronic fatigue syn- drome were not clinically depressed. Although they rated themselves as more depressed than controls on the hospital anxiety and depression scale, this did not fall within the pathological range and we could find no correlation between neuropsychological performance and ratings of depression. A recent study of depression, using the same CANTAB tests, has found a different profile of results to ours (Elliott, Sahakian, Robbins, McKay, Herrod, and Paykel, unpublished observations). Relative to their own control groups, patients with chronic fatigue syndrome were more impaired than depressed patients on the spatial working memory task and the attentional set shifting task but better at simultaneous and delayed matching to sample, planning, and spatial and pattern recognition memory tasks. Thus there is little evidence from these parallel studies to support the contention that the cognitive deficits in chronic fatigue syndrome reflect a depressive illness.

Other studies have also found some memory dysfunction suggestive of impaired cognitive effort in chronic fatigue syndrome. ${ }^{212}$ However, the deficits in these studies were more mild than ours. Furthermore, a study of patients with chronic fatigue syndrome recruited in a similar manner to ours has found no evidence of cognitive impairment on a battery of tests which included word association learning and verbal fluency. ${ }^{6}$ Similarly, Scheffers and colleagues ${ }^{15}$ have found little impairment on a task measuring event related potentials during the performance of a task which required maintenance of up to four items in short term memory while attending selectively to a relevant stimulus dimension. Thus patients with chronic fatigue syndrome were able to focus and allocate attentional resources effectively in this study. Clearly, further studies of chronic fatigue syndrome are required to explain these discrepencies. For example, a major difference between the working memory tasks in this study and ours is that ours required the subject to generate the solution rather than to follow a procedure. It would therefore be of interest to examine event related potentials in chronic fatigue syndrome during the perfomance of tasks requiring the self ordered generation of responses from memory.

We are grateful to Trevor Robbins and Barbara Sahakian for discussion and Trudy Chalder for assistance. The study was supported by a grant from The Linbury Trust.

1 Altay HT, Abbey SE, Toner BB, Salit IE, Brooker H, Garfinkel PE. The neuropsychological dimensions of postinfectious neuromyasthenia (chronic fatigue syndrome): a preliminary report. Int $\mathcal{F}$ Psychiatry Med 1990; 20:141-9.

2 Grafman J, Schwartz V, Dale JK, Scheffers M, Houser C, Straus SE. Analysis of neuropsychological functioning in patients with chronic fatigue syndrome. I Neurol Neurosurg Psychiatry 1993;56:684-9.

3 Ray C, Phillips L, Weir WRC. Quality of attention in chronic fatigue syndrome: subjective reports of everyday attention and cognitive difficulty, and performance on tasks of focused attention. Br $\mathcal{F}$ Clin Psychol 1993;32: tasks of

4 Krupp LB, Sliwinski M, Masur DM, Friedberg F, Coyle $\mathrm{PK}$. Cognitive functioning in depression in patients with chronic fatigue syndrome and multiple sclerosis. Arch chronic fatigue syndrom
Neurol 1994;51:705-10. 
5 Schmaling KB, DiClementi JD, Munro Cullum PDC, Jones JF. Cognitive functioning in chronic fatigue syndrome and depression: a preliminary comparison Psychosomatic Med 1994;56:383-8.

6 Cope H, Pernet A, Kendall B, David A. Cognitive functioning and magnetic resonance imaging in chronic fatigue. Br $\mathcal{F}$ Psychiatry 1995;167:1-9.

7 Riccio M, Thompson C, Wilson B, Morgan DJR, Lant AF Neuropsychological and psychiatric abnormalities in myalgic encephalomyelitis: a preliminary report. $\mathrm{Br} f \mathrm{Clin}$ Psychol 1992;31:111-20.

8 DeLuca J, Johnson SK, Natelson BH. Information processing efficiency in chronic fatigue syndrome and multiple sclerosis. Arch Neurol 1993;50:301-4.

9 De Luca J, Johnson SK, Beldowicz D, Natelson BH. Neuropsychological impairment in chronic fatigue syndrome, multiple sclerosis and depr

10 Smith AP. Chronic fatigue syndrome and performance. In: Smith AP, Jones DM, eds. Handbook of human performance. Vol 2. London: Academic Press, 1992:261-78.

11 Smith AP, Behan PO, Bell W, Millar K, Bakheit M Behavioural problems associated with chronic fatigue Behavioural problems associated with
syndrome. Br $\mathcal{F}$ Psychol 1993;84:41 1-23.

12 McDonald E, Cope H, David A. Cognitive impairment in patients with chronic fatigue: a preliminary study. $\mathscr{f}$ Neurol Neurosurg Psychiatry 1993;56:812-5.

13 Sandman CA, Barron JL, Nackoul K, Goldstein J, Fidler F. Memory deficits associated with chronic fatigue immune dysfunction syndrome. Biol Psychiatry 1993, 33:618-23.

14 Prasher D, Smith A, Findley L. Sensory and cognitive event related potentials in myalgic encephalomyelitis. $\mathcal{F}$ Neurol Neurosurg Psychiatry 1990;53:253-57.

15 Scheffers MK, Johnson R, Grafman J, Dale JK, Straus SE Attention and short-term memory in chronic fatigue synAttention and short-term memory in chronic fatigue syn-
drome patients: an event related potential analysis. drome patients: an event
Neurology 1992;42:1667-75.

16 Sahakian B, Owen A. Computerised assessment in neuropsychiatry using CANTAB. $\mathcal{F} R$ Soc Med 1992;85 399-402.

17 Pawlikowska T, Chalder T, Hirsch S, Wallace P, Wright D, Wessely $S$. A population based study of fatigue and psychological distress. BMF 1994;308:743-6.

18 Wessely S, Chalder T, Hirsch S, Pawlikoska T, Wallace P, Wright D. Post infectious fatigue: a prospective cohor study in primary care. Lancet 1996 (in press).

19 Chalder T, Berelowitz G, Pawlikowska T, et al. Development of a fatigue scale. $\mathcal{A}$ Psychosom Res 1993;37:147-53.

20 Lewis G, Pelosi A, Araya R, Dunn G. Measuring psychiatric disorder in the community: a standardised assess-
ment for lay interviewer. Psychol Med 1992;22:465-86.

21 Sharpe $M$, Archard L, Banatvala J, et al. Chronic fatigue syndrome: guidelines for research. $f R$ Soc Med 1991, 84:118-21.

22 Nelson $\mathrm{HE}$ and Willison, J. The National adult reading test. Test manual. 2nd ed. Windsor: NFER-Nelson, 1992.

23 Zigmond AS, Snaith RP. The hospital anxiety and depression scale. Acta Psychiatrica Scand 1984;67:361-70.

24 Milner B. Interhemispheric differences in the localisation of psychological processes in man. Br Med Bull 1971;27: 272-7.

25 Shallice T. Specific impairments in planning. Philos Trans $R$ Soc Lond Biol 1982;298:199-209.

26 Wechsler D. Wechsler memory scale revised. London: The Psychological Corporation, 1987.

27 Kullback S. Information theory and statistics. New York:
John Wiley, 1959.

28 Robbins TW. A critique of the methods available for the measurement of spontaneous motor activity. In: Iversen LL, Iversen SD, Snyder SH, eds. Handbook of psychopharmacology. Vol 7. New York: Plenum Press, 1977: 37-82.

29 Owen A, Downes J, Sahakian B, Polkey C, Robbins T. Planning and spatial working memory following fronta

30 Joyce E, Robbins T. Frontal lobe function in Korsakoff and non-Korsakoff alcoholics: planning and spatial working memory. Neuropsychologia 1991;29:709-23.

31 Owen A, James M, Leigh P, et al. Fronto-striatal cognitive deficits at different stages of Parkinson's disease. Brain 1992;115:1727-51.

32 Robbins TW, James M, Lange KW, Owen AM, Quinn NP, Marsden CD. Cognitive performance in multiple system atrophy. Brain 1992;115:271-91.

33 Robbins TW, James M, Owen AM, et al. Cognitive deficits in progressive supranuclear palsy, Parkinson's disease and multiple system atrophy in tests sensitive to frontal lobe dysfunction. $\mathcal{F}$ Neurol Neurosurg Psychiatry 1994;57: 79-88.

34 Robbins TW, James M, Owen AM, McKinnes L, Rabbitt P. Cambridge automated neuropsychological test battery (CANTAB). A factor analytic study in a large sample of normal volunteers. Dementia 1994;5:266-81.

35 Joyce EM, Robbins TW. Cognitive deficits in Korsakoff and non-Korsakoff alcoholics following alcohol withdrawal and their relationship to length of abstinence. Alcohol and Alcoholism Supplement 1993;2:501-5.

36 Sahakian B, Morris R, Evenden J, et al. A comparative study of visuospatial memory and learning in Alzheimertype dementia and Parkinsons's disease. Brain 1988; 111:695-718.

37 Sahgal A, Sahakian BJ, Robbins TW, et al. Detection of visual memory and learning deficts in Alzheimer's disease visual memory and learning deficts in Alzheimer's disease
using the Cambridge neuropsychological test automated using the Cambridge neuropsych

38 Galloway PH, Sahgal A, McKeith IG, et al. Visual pattern recognition memory and learning deficits in senile dementias of Alzheimer and Lewy body type. Dementia 1992;3:101-3

39 Sahgal A, Lloyd S, Wray CJ, et al. Does visuospatial memory in Alzheimer's disease depend on the severity of the disorder? International fournal of Geriatric Psychiatry 1992; 7:427-36.

40 Owen AM, Sahakian BJ, Semple J, Polkey CE, Robbins TW. Visuospatial short term recognition memory and learning after temporal lobe excisions, frontal lobe excisions or amygdalo-hippocampectomy in man. Neuropsychologia 1995;33:1-24.

41 Hasher L, Zacks RT. Automatic and effortful processes in memory. $\mathcal{F}$ Exp Psychol 1979;3:356-88.

42 Cohen RM Weingrtner $H$, Smallberg SA, Pickar D Murphy DL. Effort and cognition in depression. Arch Gen Psychiatry 1982;39:593-7.

43 Roy-Byrne PP, Weingartner H, Bierer LM, Thompson K, Post RM. Effortful and automatic processes in depression. Arch Gen Psychiatry 1986;43:265-67.

44 Robbins TW, Joyce EM, Sahakian BJ. Neuropsychology and neuroimaging of affective disorders. In: Paykel E, ed. Handbook of affective disorders. London: Churchill Livingstone, 1992:

45 O'Brien JT, Sahakian BJ, Checkley SA. Cognitive impairments in patients with seasonal affective disorder. $\mathrm{Br} \mathcal{F}$ Psychiatry 1993;163:338-43. 